\title{
Ocorrência de contusões em carcaças bovinas em frigorífico
}

\author{
Bruises occurrence in cattle carcasses at a slaughterhouse
}

\author{
PETRONI, Rudge ${ }^{*}$; BÜRGER, Karina Paes ${ }^{2}$; GONÇALEZ, Patrícia Oliveira ${ }^{3}$; ROSSI, \\ Gabriel Augusto Marques ${ }^{4}$; VIDAL-MARTINS, Ana Maria Centola ${ }^{5}$; AGUILAR, \\ Carlos Eduardo Gamero ${ }^{4}$
}

${ }^{\mathbf{1} M e ́ d i c o ~ V e t e r i n a ́ r i o, ~ A u t o ̂ n o m o, ~ S a ̃ o ~ P a u l o, ~ B r a s i l . ~}$

${ }^{2}$ Universidade Estadual Paulista, Faculdade de Ciências Agrárias e Veterinárias, Departamento de Medicina Veterinária Preventiva e Reprodução Animal, Jaboticabal, São Paulo, Brasil.

${ }^{3}$ Universidade Camilo Castelo Banco, Descalvado, São Paulo, Brasil.

${ }^{4}$ Universidade Estadual Paulista, Faculdade de Ciências Agrárias e Veterinárias, Programa de PósGraduação de Medicina Veterinária, Jaboticabal, São Paulo, Brasil.

${ }^{5}$ Universidade de São Paulo, Faculdade de Zootecnia e Engenharia de Alimentos, Departamento de Medicina Veterinária, Pirassununga, São Paulo, Brasil.

*Endereço para correspondência: rudge_petroni@hotmail.com

\section{RESUMO}

O bem-estar animal é uma prática que implica na manutenção das liberdades psicológicas e fisiológicas dos animais, para que seus organismos permaneçam saudáveis até o abate, proporcionando uma carcaça com qualidade e evitando sofrimentos desnecessários aos mesmos. Uma forma de avaliar o bem-estar é através da monitoração da ocorrência de contusões nas carcaças, um indicativo de manejo inadequado dos mesmos. Objetivou-se avaliar a ocorrência de contusões em carcaças bovinas em um matadouro-frigorífico localizado na Região Centro-oeste do Estado de São Paulo. Assim, foi aplicada "check-list", para determinação da presença de contusões, o local e a gravidade das mesmas, classificando-as entre escore I a III. Do total de animais avaliados, $98 \% \quad(880 / 898) \quad$ apresentaram contusão. As contusões de grau I na região do coxão das meias carcaças tiveram maior incidência com média de $61,8 \%$ entre os lados direito e esquerdo. Também foram observadas múltiplas lesões em diversos animais abatidos. Em 133 animais avaliados foram retirados 15,6 $\mathrm{kg}$ de material lesionado na região do coxão apenas, demonstrando grandes prejuízos econômicos aos produtores e às indústrias por falhas em bem-estar animal. O manejo pré-abate inadequado compromete o bem-estar animal e a qualidade das carcaças, desprestigiando nossos produtos no comércio internacional da carne bovina e consistindo em perdas econômicas à pecuária bovina nacional.

Palavras-chave: abate, bem-estar animal, lesões, qualidade de carne

\section{SUMMARY}

The animal welfare is a series of practices that involves the maintenance of psychological and physiological freedoms of animals, to them remain healthy until slaughter and provide a satisfactory quality carcass and to avoid unnecessary suffering to animals. One way to evaluate the animal welfare is monitoring the occurrence of bruises on carcasses that is an indicative of inadequate management of the animals. The present study aimed to evaluate the occurrence of bruises in cattle carcasses at a slaughterhouse located in the Midwest Region of the State of São Paulo, Brazil. Was applied a "check-list" that determined the presence of bruises, the location and severity of them, ranking among scores I to III. Was observed in $98 \%(880 / 898)$ of the animals the presence of contusion. The Grade I injuries in the region of the hips at half carcasses had higher incidence with an average of $61.8 \%$ between the right and left sides. Could be observed multiple lesions in several animals slaughtered. In 133 animals 
observed were removed 15.6 kilograms of material only the injured region only of the hips, demonstrating economic losses to cattle farmers and industries for failures in animal welfare practices. The inappropriate pre-slaughter management compromises animal welfare and the quality of carcasses, discrediting our products on international meat trade and consisting in economic losses to the national cattle herds.

Keywords: animal welfare, lesions, meat quality, slaughter

\section{INTRODUÇ̃̃O}

O tema bem-estar animal vem ganhando espaço no mercado mundial de alimentos, pois evita sofrimento desnecessário aos animais e permite a produção de alimentos com qualidade (OIE, 2009). Essas práticas de manejo que permitem o bem-estar dos animais devem estar presentes em todas as etapas que precedem e durante o abate dos animais, ou seja, desde a criação até o momento da sangria.

Schwartzkopf-Genswein et al. (2012) destacaram a importância de realizar o transporte de maneira adequada, devido as perdas sobre a qualidade da carne produzida. Essas podem ser quantitativas, devido à remoção de áreas condenadas devido à presença de hematomas e contusões, e/ou qualitativas, que ocorrem devido ao elevado valor de $\mathrm{pH}$, e consequente coloração escura da carne, elevada susceptibilidade à presença de microorganismos deteriorantes e patogênicos na carne obtida de um animal estressado. Isso ocorre devido à depleção do glicogênio muscular, o responsável pela redução do $\mathrm{pH}$ post-mortem devido à sua conversão em ácido lático (WARNER et al., 2005).

A presença de hematomas e contusões é um indicativo de um manejo inadequado (GRANDIN, 2010).
Civieira et al. (2006) observaram que as contusões nos quartos foram as mais frequentes, seguidas pelas localizadas no vazio, lombo, paleta e costelas, demonstrando perdas de cortes valorizados por falhas oriundas do mau manejo. Nascimento et. al (2009) observaram uma elevada presença de contusões (66\%) nos animais avaliados, detectando observando com maior frequência nas fêmeas e principalmente no traseiro. Em estudo semelhante, Peñuela et al. (2011) também avaliaram a ocorrência de contusões em carcaças bovinas, encontrando $84,3 \%$ das carcaças contundidas, observado uma maior ocorrência nas fêmeas também, concordando com o observado por Nascimento et al. (2009).

Em estudo realizado por Willian et al. (2012), foi avaliada a taxa de hematomas em bovinos transportados em diferentes tipos de caminhão. Observaram diferença quanto a frequência de ocorrência de acordo com o tipo de caminhão utilizado e a distância percorrida, destacando a grande influência do modo de transporte sobre a qualidade da carcaça dos animais.

Devido as perdas econômicas na cadeia produtiva da carne bovina, relacionadas ao manejo inadequado, que não atende ao bem estar animal, objetivou-se avaliar a ocorrência de contusões e sua distribuição em carcaças bovinas de um matadouro-frigorífico.

\section{MATERIAL E MÉTODOS}

Este trabalho foi desenvolvido em frigorífico sob registro e supervisão do Serviço de Inspeção Federal (S.I.F) da Região Centro-Oeste do Estado de São Paulo, com média de abate de 900 animais por dia e cerca de 100 animais por hora. A avaliação da presença de 
contusões foi realizada em número significativo e proporcional ao tamanho dos lotes, totalizando 898 animais. Nos lotes menores que 50 animais, foram incluídos todos os animais, e em lotes acima desse valor, utilizava-se um mínimo de $25 \%$ do tamanho do lote.

A avaliação da presença de contusões foi visual, aproveitando as etapas de inspeção correspondentes às linhas $\mathrm{H}$ e I (inspeção interna e externa das partes caudal e cranial das carcaças). $\mathrm{O}$ registro das ocorrências foi por meio do formulário que contemplava a procedência dos animais, a distância desde a origem até $\mathrm{o}$ frigorífico, a localização da lesão e o grau (intensidade) da contusão nos animais, todos preenchidos na forma de checklist (adaptado de ALMEIDA, 2005).

A localização da lesão foi determinada por meio da divisão mediana das carcaças, posteriormente, por meio de uma divisão imaginária desta em áreas como: coxão, lombo, gradil costal e dianteiro, essa utilizadas como cortes comerciais em estabelecimentos produtores de carnes.

O grau de severidade da contusão (ou hematoma) foi determinado de acordo com a classificação de carcaças, variando de ausente até grau III. Animais classificados como ausente não apresentam contusões; grau I, para lesões que acometem os tecidos subcutâneos; grau II para lesões mais profundas, que atingem também tecidos musculares; e grau III para aquelas que apresentam tecido ósseo comprometido (adaptado de ALMEIDA, 2005).

A determinação das características de ocorrência das contusões foram avaliadas sendo estabelecidas as frequências de ocorrência, ou seja, a porcentagem de partes acometidas de acordo com determinadas características em relação ao total das avaliadas.

\section{RESULTADOS E DISCUSSÃO}

Foi observado que as frequências de lesões foram mais baixas em distâncias menores que $200 \mathrm{~km}$, em que se observou a ocorrência média de 2,53 hematomas por animal abatido (Tabela1). Aumentaram para 3,37 lesões por animal quando a distância passava para 201 a $400 \mathrm{~km}$, e ainda quando se ultrapassava os $401 \mathrm{~km}$ aumentaram em para 3,83 lesões. Esse aumento gradativo, visualizado nessa diferença de espaço percorrido, mostra que a distância do transporte pode afetar diretamente a ocorrência de contusões.

Tabela 1. Distância de transporte percorrida do local de origem até o estabelecimento de abate e ocorrência de lesões nas carcaças em um frigorífico da Região Centro-oeste do Estado de São Paulo, em 2008

\begin{tabular}{cccc}
\hline Distância $(\mathrm{km})$ & Número de lotes & Número de animais & Lesões \\
\hline$<200$ & 5 & 211 & 535 \\
201 a 400 & 11 & 299 & 1009 \\
$>401$ & 8 & 388 & 1488 \\
\hline Total & 24 & 898 & 3032 \\
\hline
\end{tabular}

Tal achado ser explicado pela maior possibilidade de práticas bruscas durante a direção do véiculo de acordo com a duração da viagem, pelas 
condiçoes do asfalto, ou ainda, por uma maior velocidade observada durante o percurso. Tais dados servem de apoio à implantação de programas de autocontrole relacionados ao bem-estar animal dentro dos abatedouros de bovinos, permitindo categorizar os produtores que necessitam de maior apoio para a implantação desses programas devido a um maior risco determinado.

Nos 898 animais avaliados houve predomínio de contusões na hemicarcaça esquerda. $\mathrm{O}$ número de contusões foi maior do lado direito somente para o coxão, como pode ser visto na Tabela 2 , que mostra as quantidades totais de contusões encontradas. Tal fato pode ser atribuído às práticas de manejo serem realizadas com maior frequência no lado esquerdo dos animais, devido à disposição que as estruturas são construídas dentro da indústria. Isso permite considerar que falhas de bemestar animal ocorrem dentro da indústria também, além das possíveis ocorridas durante as etapas de produção e transporte.

Tabela 2. Quantidade de lesões encontradas nos animais abatidos em um frigorífico da Região Centro-oeste do Estado de São Paulo de acordo com a sua localização, em 2008

\begin{tabular}{lccccc}
\hline Local & Coxão & Lombo & Gradil costal & Dianteiro & Total \\
\hline Hemicarcaça esquerda & 970 & 103 & 374 & 154 & 1601 \\
Hemicarcaça direita & 974 & 84 & 290 & 83 & 1431 \\
\hline Total & 1944 & 187 & 664 & 237 & 3032 \\
\hline
\end{tabular}

A quantidade de lesões observadas em 880 animais foi de 3032, ou seja, em média 3,37 por carcaça avaliada. Esse dado pode ser comparado com Civieira et al. (2006) que verificaram que a maior prevalência de lesões situa-se entre duas a quatro por carcaça, concordando com esse trabalho.

As contusões foram estudadas quanto à gravidade nas carcaças. Como se pode observar na Tabela 3, não foram observadas lesões grau III, pois quando ocorre esse tipo de lesão é realizado o abate de emergência, que é realizado em matadouro de emergência adjacente a planta frigorífica em que se realizou o presente trabalho, não entrando assim, nos dados coletados.

Tabela 3.Frequência da localização das contusões em um frigorífico do Estado de São Paulo em 2008

\begin{tabular}{lcccc}
\hline Localização da lesão & \multicolumn{2}{c}{ Esquerda } & \multicolumn{2}{c}{ Direita } \\
\hline Grau & 1 & 2 & 1 & 2 \\
\hline Coxão & $59,8 \%$ & $36,19 \%$ & $63,81 \%$ & $34,97 \%$ \\
Lombo & $7,461 \%$ & $4,009 \%$ & $5,902 \%$ & $3,452 \%$ \\
\hline Gradil Costal & $32,63 \%$ & $8,575 \%$ & $25,17 \%$ & $6,347 \%$ \\
Dianteiro & $12,92 \%$ & $4,009 \%$ & $6,793 \%$ & $2,45 \%$ \\
\hline
\end{tabular}


Dos 898 animais avaliados, apenas 18 (2\%) não apresentaram nenhum tipo de lesão, 39 (4,34\%) animais não apresentaram lesões na hemicarcaça esquerda e $56(6,23 \%)$ animais não apresentaram na direita.

Esses valores de ocorrência (98\%) das carcaças avaliadas são superiores ao encontrado por Nascimento et. al (2009) no Estado do Pará e Peñuela et al. (2011) na Colômbia, que observaram a presença de contusões em $66 \%$ e $84,3 \%$ dos animais avaliados, respectivamente, demonstrando que a indústria avaliada possui falhas em gestão de bem-estar animal e perda da qualidade da carne, necessitando capacitar os pecuaristas fornecedores, transportadores e todos os responsáveis pelo manejo dos animais. Em estudo realizado por Willian et al. (2012), foi encontrado grande número de lesões no traseiro e contrafilé, igualmente observado nesse estudo, demonstrando perdas econômicas oriundas de cortes valorizados comprometidos.

Também se pode observar múltiplas $(2$ ou 3) lesões no mesmo local, em ambos os lados, principalmente na região do coxão, como especificados na Tabela 4 , sendo resultadas do mau manejo realizado com os animais.

Tabela 4. Número de animais que apresentaram duas ou três lesões no mesmo local, em um frigorífico no Estado de São Paulo, em 2008.

\begin{tabular}{lcccccccc}
\hline & \multicolumn{3}{c}{ Esquerdo } & \multicolumn{3}{c}{ Direito } \\
\cline { 2 - 9 } Numero de lesões & \multicolumn{3}{c}{ Grau 1 } & \multicolumn{2}{c}{ Grau 2 } & Grau 1 & Grau 2 \\
\cline { 2 - 9 } & 2 & 3 & 2 & 3 & 2 & 3 & 2 & 3 \\
\hline Coxão & 60 & 2 & 54 & 3 & 45 & 1 & 30 & 0 \\
Lombo & 0 & 0 & 0 & 0 & 0 & 0 & 0 & 0 \\
Gradil & 2 & 0 & 6 & 0 & 2 & 0 & 1 & 0 \\
Dianteiro & 2 & 0 & 0 & 0 & 0 & 0 & 0 & 0 \\
\hline
\end{tabular}

As contusões de grau I na região do coxão das meias carcaças tiveram maior incidência com média de $61,8 \%$ entre os lados, resultado mais alto, porém compatíveis, devido ao fato que a região posterior teve maior incidência. Compatíveis com Civieira et al. (2006), que na média da incidência entre o lado direito e lado esquerdo foi de 55\%. As lesões na região do lombo, considerando os lados direito $\mathrm{e}$ esquerdo e os graus I e II, apresentaram uma média de 5,21\%. Os mesmos autores encontraram 11,8\%, valor praticamente mais que o dobro do encontrado no presente trabalho. A maior ocorrência nessas regiões pode ser atribuída ao fato dos animais serem movimentados com $\mathrm{o}$ funcionário localizado na parte traseira ou mediana do animal, para que o mesmo avance para frente, e aumentando a possibilidade de impactos e utilização de equipamentos indevidos na região traseira.

Como a quantidade de contusões que ocorrem no coxão é alta, e nele se localizam os cortes nobres da carcaça, foi pesada a porção retirada na toalete de 133 animais, sendo totalizada uma perda de $15,6 \mathrm{~kg}$, que equivale $117,3 \mathrm{~g}$ retirados por animal. Considerando o valor da arroba $\mathrm{R} \$ 90,00$ por animal e perda de $117,3 \mathrm{~g}$, o prejuízo por animal seria de $R \$ 0,70$ ao pecuarista somente pelas lesões no coxão, representando perda pequena, porém significativa devido ao fato de as lesões poderem 
estar localizadas em outras regiões e com maior severidade também, que consistiria em múltiplas perdas aos produtores. Considerando, ainda, que no estabelecimento observado são abatidos em média 19.800 animais, o prejuízo mensal, apenas da toalete do coxão, seria de $\mathrm{R} \$ 13.934,40$, demonstrando perdas econômicas nessa cadeia produtiva devido a falhas em bem-estar animal.

O grande número de lesões encontradas evidencia falhas em bem-estar animal em um abatedouro brasileiro de bovinos, podendo ser oriundas de produtores ainda não capacitados quanto ao tema e que ainda praticam um manejo inadequado aos animais; de falhas nas etapas de embarque, transporte e desembarque, realizados de maneira inadequada; na etapa de condução dos animais à sala de matança ou realização de insensibilização inadequada. Ainda, a ausência de um programa de bem-estar animal devidamente implantado, acarreta desprestígio no mercado internacional de carnes e enormes perdas diretas para a cadeia produtiva da carne bovina devido à remoção de áreas acometidas por lesões oriundas do mau manejo. São necessárias melhorias a implantanção de programas de bem-estar animal dentro das indústrias processadoras da carne bovina, e também na fiscalização dos mesmos pelos órgãos responsáveis, para a consolidação dessa cadeia produtiva brasileira no mercado mundial.

\section{REFERÊNCIAS}

\author{
ALMEIDA, L.A.M. Manejo no pré- \\ abate de bovinos: aspectos \\ comportamentais e perdas \\ econômicas por contusões. 2005. 62p. \\ Dissertação (Mestrado em Medicina
}

Veterinária) - Faculdade de Ciências Agrárias e Veterinárias, Universidade Estadual Paulista, Jaboticabal.

CIVIEIRA, M.P.; RENNER, R.M.; RODRIGUES, N.C.; VARGAS, R.E.S.; Avaliação do bem-estar animal em bovinos abatidos para consumo em frigorífico do Rio Grande do Sul.

Revista Veterinária em Foco, v.4 n.1 p.5-11, 2006.

GRANDIN, T. Auditing animal welfare at slaughter plants. Meat Science, v.86, p.56-65, 2010.

NASCIMENTO, G.R.; RODRIGUES, W.B.; MARTINS, N.E.X.; DIAS, F.E.F.; CAVALCANTE, T.V.; FERITAS, F.L.C.; LEIRA, M.H.; ALMEIDA, K. de S. Avaliação do bemestar animal em bovinos abatidos no Pará. Revista Veterinária em Foco, v.6, n.2, p.121-127, 2009.

\section{WORLD ORGANISATION FOR} ANIMAL HEALTH - OIE. Killing Animals for Disease Control Purposes, Terrestrial Animal Health Code. 18th Edition. Paris, France, 2009.

PEÑUELA, M.H.R.; TORO, C.G.; VALENCIA, J.A.S. Evaluación del manejo presacrificio y su relación com la presencia de contusiones em canales bovinas. Biosalud, v.10, n.2, p.28-36, 2011.

SCHWARTZKOPF-GENSWEIN, K.S.; FAUCITANO, L.; DADGAR, S.; SHAND, P.; GONZÁLEZ, L.A.; CROWE, T. G. Road transport of cattle, swine and poultry in North America and its impact on animal welfare, carcass and meat quality: a review. Meat Science, v.92, p.227-243, 2012. 
WARNER, R.D., FERGUSON, D.M., MCDONAGH, M.B., CHANNON, H.A., COTTRELL, J.J.; DUNSHEA,

F.R. Acute exercise stress and electrical stimulation influence the consumer perception of sheep meat eating quality and objective quality traits. Australian Journal of Experimental Agriculture, v.45, p.553-560, 2005.

WILLIAM, B.; SILVA, J.L. da; ABREU, J.S. de.; ANDREOLLA, D.L. Bem estar e taxa de hematomas de bovinos transportados em diferentes distâncias e modelos de carroceria no estado do Mato Grosso do Sul - Brasil. Revista Brasileira de Saúde Pública e Produção Animal [online], v.13, n.3, p.850-859, 2012.

Data de recebimento: 10/06/2013

Data de aprovação: 09/09/2013 